\title{
Increased efficiency of low band gap polymer solar cells at elevated temperature and its origins
}

\author{
Bin Yang, ${ }^{1,2}$ James Cox, ${ }^{1}$ Yongbo Yuan, ${ }^{1,2}$ Fawen Guo, ${ }^{1,2}$ and Jinsong Huang ${ }^{1,2, a)}$ \\ ${ }^{1}$ Department of Mechanical Engineering, University of Nebraska-Lincoln, Lincoln, Nebraska 68588, USA \\ ${ }^{2}$ Nebraska Center for Materials and Nanoscience, Lincoln, Nebraska 68583, USA
}

(Received 7 June 2011; accepted 31 August 2011; published online 30 September 2011)

\begin{abstract}
Photovoltaic characteristics of a low bandgap polymer, poly[(4,4'-bis(2-lethylhexyl)dithieno[3,2-b:2', 3'-d]silole)-2,6-diyl-alt-(2,1,3-benzothiadiazole)-4,7-diyl], based bulk hetero-junction organic photovoltaic were investigated from room temperature (RT) to $145^{\circ} \mathrm{C}$ to evaluate its applications in harsh environments. The power conversion efficiency was found to increase from $4.1 \%$ at RT to $4.5 \%$ at $105^{\circ} \mathrm{C}$ with increased short circuit current density $\left(J_{s c}\right)$ and fill factor $(F F)$ despite the decreased open circuit voltage $\left(V_{o c}\right)$. Detailed investigation revealed that $J_{s c}$ and $F F$ improvements were caused by the increased and balanced carrier mobilities at higher temperatures. The $V_{o c}$ of the low bandgap polymer solar cell is determined not only by the energy levels and dark currents, but also by the binding energy of charge transfer excitons (CTEs). A slower reduction of $V_{o c}$ is observed at high temperatures due to the decreased binding energy of CTEs. (C) 2011 American Institute of Physics. [doi:10.1063/1.3643450]
\end{abstract}

Bulk heterojunction organic photovoltaics (BHJ-OPVs) are among the most promising candidates to harvest energy in large scales and at low cost. Recently, the high power conversion efficiency (PCE) within the range of 6\%-9\% has been achieved with synthesized materials and device processes. ${ }^{1-3}$ The BHJ-OPVs products from Konarka have already demonstrated an excess of five years roof-top stability. In the light of these advances in PCE and stability improvement in BHJ-OPVs, it is time to seriously consider their application in all kinds of conditions, including harsh environments such as high temperature deserts. However, unlike the model polymers such as poly-3(hexylthiophene), the temperature dependent performance of low bandgap polymer, which is more likely to be a commercialized product, has not yet been explored.

This manuscript reports the temperature dependent performance of low bandgap polymer solar cells based on poly[ $\left(4,4^{\prime}\right.$-bis(2-lethylhexyl)dithieno[3,2-b:2', $3^{\prime}$-d $]$ silole)2,6-diyl-alt-(2,1,3-benzothiadiazole)-4,7-diyl] (PSBTBT). The BHJ PSBTBT:[6,6]-phenyl-C61-butyric acid methyl ester (PCBM) devices were fabricated following the method reported elsewhere with a blending ratio of $1: 1$ by weight, ${ }^{4,5}$ and device structure of indium tin oxide (ITO)/poly(3,4-ethylenedioxythiophene):poly(styrenesulfonate) (PEDOT:PSS) (35 nm)/PSBTBT:PCBM (80 nm)/calcium (Ca) $(20 \mathrm{~nm}) / \mathrm{alu}-$ minum $(\mathrm{Al})(100 \mathrm{~nm})^{2}$ This test was done in air under simulated illumination of Air Mass 1.5 Global solar irradiation. The PCE of these devices is around $4.1 \%$ at room temperature (RT), which is consistent with others reported. ${ }^{2}$ The temperature dependent photocurrent density-voltage curves were measured at temperatures from RT to $145^{\circ} \mathrm{C}$ as shown in Fig. 1(a). The variation of short circuit current density $\left(J_{s c}\right)$, open circuit voltage $\left(V_{o c}\right)$, fill factor $(F F)$, and PCE were summarized in Fig. 1(b). When the operation temperature increases, both $J_{s c}$ and $F F$ keep increasing while $V_{o c}$

${ }^{a)}$ Electronic mail: jhuang2@unl.edu. Telephone: (402) 472-2640. decreases. Before reaching $105^{\circ} \mathrm{C}$, the increasing of $J_{s c}$ and $F F$ overrides the decreasing $V_{o c}$ and eventually results in a maximum PCE of $4.5 \%$ at $105^{\circ} \mathrm{C}$, which is $10 \%$ higher than that at RT. In order to verify whether morphological change due to thermal annealing contributes to the increased efficiency, the devices have been annealed at a temperature of $125^{\circ} \mathrm{C}$ for 20 minutes before the $J-V$ curve measurement. The photocurrents measured after 10 minutes annealing and 20 minutes annealing show negligible difference, which are also shown in the inset of Fig. 1(a). Therefore, the efficiency improvement of the PSBTBT:PCBM BHJ-OPVs at high temperatures should be ascribed to temperature dependence of carrier dynamics instead of morphological change of the polymer blends. It is of great importance to understand the carrier dynamics at high temperatures from the point of view of fundamental understanding of device physics, especially the origin of the $V_{o c}$ in BHJ-OPVs.

The $J_{s c}$ is a function of several BHJ-OPVs device properties: light absorption, carrier mobility, and carrier recombination. ${ }^{6}$ The absorption spectrum of the PSBTBT:PCBM film was found to decrease slightly (around $0.7 \%$ ) at $105^{\circ} \mathrm{C}$. Therefore, the increase of $J_{s c}$ at high temperatures must originate from other factors. The carrier mobility of the PSBTBT:PCBM film was measured at different temperatures. The electron-only current and hole-only current of the PSBTBT:PCBM film were measured and fitted by the space charge limited current model. ${ }^{7}$ The structure of hole-only devices is ITO/PEDOT:PSS/ PSBTBT:PCBM/molybdenum oxide $\left.\left(\mathrm{MoO}_{3}\right) / \mathrm{Al}\right)$, and the structure of electron-only devices is ITO/cesium carbonate $\left(\mathrm{Cs}_{2} \mathrm{CO}_{3}\right) / \mathrm{PSBTBT}: \mathrm{PCBM} / \mathrm{Cs}_{2} \mathrm{CO}_{3} / \mathrm{Al}$. The spin-coated and thermal evaporated $\mathrm{Cs}_{2} \mathrm{CO}_{3}$ can leave a low work function surface for electron-only devices. ${ }^{8}$ As shown in Fig. 2, both electron and hole mobility increase with temperature, which can be explained by the thermally activated carrier transport. The hole mobility increased by 16 times at $145^{\circ} \mathrm{C}$, while the electron mobility only enhanced by seven times compared to those at RT. As a result, electron/hole mobility ratio decreased from 6.4 

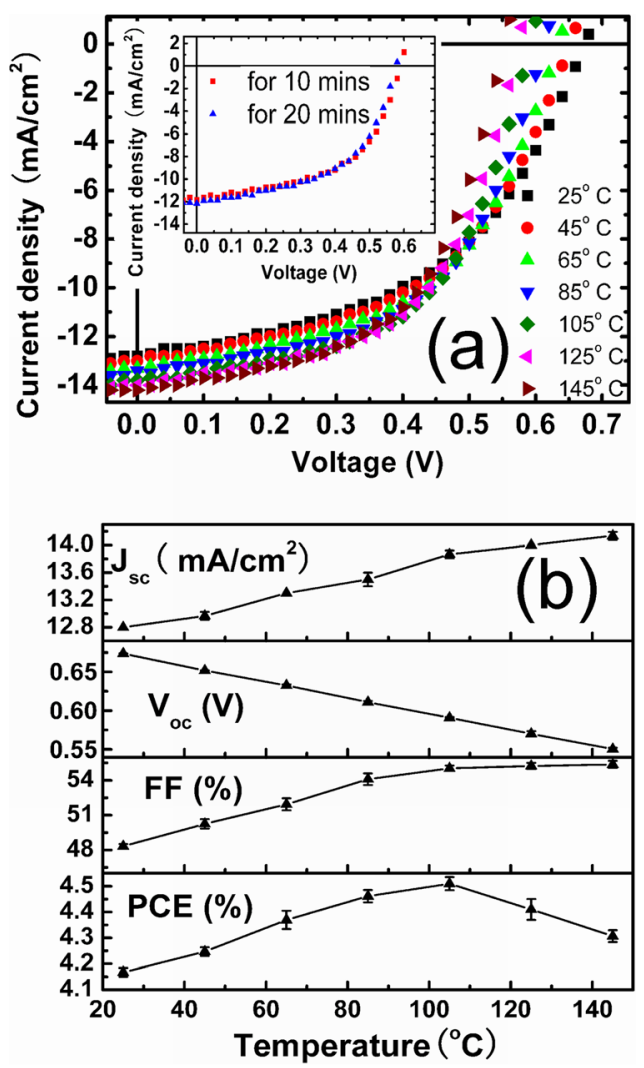

FIG. 1. (Color online) (a) Variation of $J$-V curve with temperature. The inset shows the change of $J-V$ curve at $125^{\circ} \mathrm{C}$ between after annealing 10 minutes and annealing 20 minutes; (b) Variation of photovoltaic parameters with temperature from RT to $145^{\circ} \mathrm{C}$.

at RT to 2.8 at $145^{\circ} \mathrm{C}$. This better balanced electron and hole mobility, together with the higher carrier mobilities at high temperatures, resulted in higher charge carrier extraction efficiency, and thus, a higher $J_{s c}$.

The origin of $V_{o c}$ in BHJ-OPVs remains controversial. Previous studies have generally suggested that the $V_{o c}$ of $\mathrm{BHJ}-$ OPVs is well correlated to the energy difference between the highest occupied molecular orbit level (HOMO) of the donor and the lowest unoccupied molecular orbit level (LUMO) of the acceptor. ${ }^{1,9}$ The $V_{o c}$ is also strongly influenced by other factors such as carrier recombination ${ }^{3,10}$ and shunt resistance. ${ }^{11}$ $V_{o c}$ reduces with a decreased shunt resistance (by current leaking) or increased carrier recombination. Both of them result in an increased reverse bias saturated dark current. Hence, the $V_{o c}$

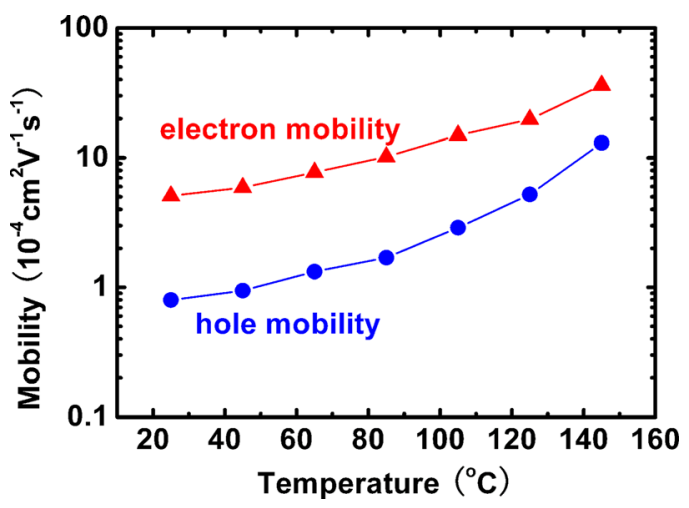

FIG. 2. (Color online) Variation of carrier mobilities with temperature. variation could often be inferred from the reverse bias saturated dark current. Temperature dependant $V_{o c}$ variation provides an excellent opportunity to understand the origin of $V_{o c}$ in the low bandgap polymer BHJ-OPVs.

As shown in Fig. 1(b), $V_{o c}$ decreased almost linearly with increasing temperature for PSBTBT:PCBM BHJ-OPVs. In order to determine whether the increased reverse bias saturated dark current at high temperatures solely causes the reduced $V_{o c}$, we used the following equation to estimate the contribution from the reverse bias saturated dark current variation to the observed reduced $V_{o c}:^{12}$

$$
V_{o c}^{T}=V_{o c}^{R T}-\left[n^{R T} \frac{300 k}{q} \ln \left(\frac{J_{s c}^{R T}}{J_{o}^{R T}}\right)-n^{T} \frac{k T}{q} \ln \left(\frac{J_{s c}^{T}}{J_{o}^{T}}\right)\right],
$$

where $V_{o c}^{T}$ is the $V_{o c}$ at temperature $T, J_{s c}^{T}$ is the $J_{s c}$ at temperature $T, J_{0}^{T}$ is the reverse saturation current density at temperature $T, n^{T}$ is the diode ideality factor at temperature $T, k$ is the Boltzmann constant,and $q$ is the elemental electron charge. Both $n^{T}$ and $J_{o}^{T}$ were derived by fitting dark current density curve according to the method reported elsewhere. ${ }^{13}$ Using the $V_{o c}$ at RT as a reference, the $V_{o c}$ at elevated temperature was calculated and shown in the green triangle in Fig. 3(a). It is found that the calculated $V_{o c}$ reduces with temperature at a higher rate than the experimentally measured value. This result indicates that apart from the increased reverse bias saturated dark current, there must be other mechanism(s) that slow(s) down the reduction of $V_{o c}$ with increasing temperature.
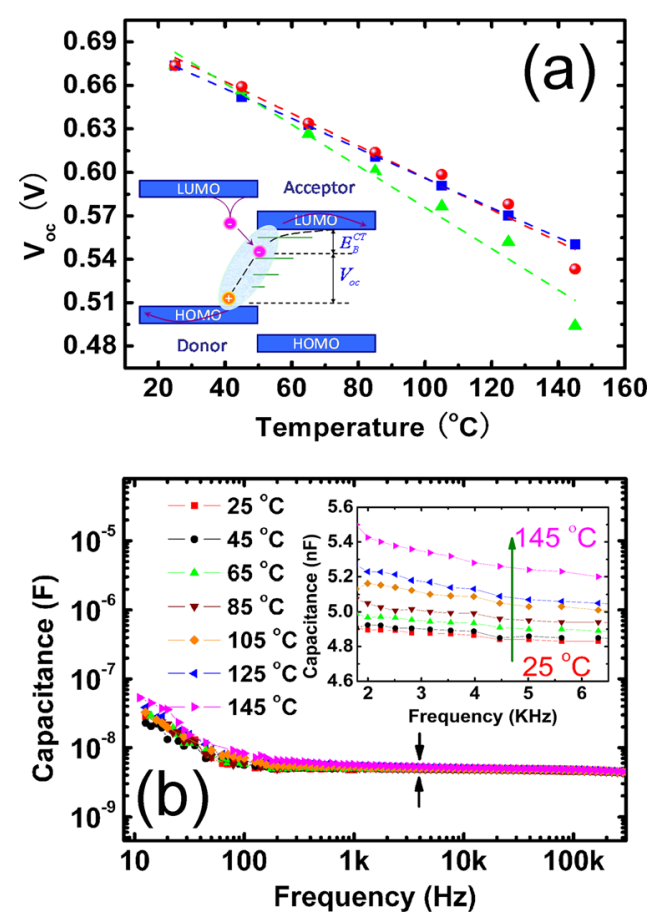

FIG. 3. (Color online) (a) Variation of $V_{o c}$ with temperature. The experimentally measured $V_{o c}$ is shown in blue solid squares. The calculated $V_{o c}$ from Eq. (1) is shown in green solid triangles. Red solid circles show the calculated $V_{o c}$ through adding the calculated $\Delta V_{o c}$ from Eq. (3) to the calculated $V_{o c}$ from Eq. (1). The inset shows the energetic level diagram of PSBTBT:PCBM interface. (b) Variation of device capacitance with frequency at different temperatures. The inset shows the increase of device capacitance with temperature at $4 \mathrm{kHz}$. 
For a better understanding of the slower reduced $V_{o c}$ at high temperatures, the variation with temperature of binding energy of the charge transfer excitons (CTE) in PSBTBT:PCBM was also studied here. In BHJ-OPVs, the CTE is formed through the attractive Coulombic force between the electron on the acceptor and the hole on the donor right after the charge transfer. The CTEs can be taken as the precursor of the free carriers, and their bandgap determines the $V_{o c}{ }^{6}$ As shown in the inset of Fig. 3(a), the bandgap $\left(E_{\mathrm{g}}\right)$ of CTE can be described by the following equation: ${ }^{6}$

$$
E_{g}=\left(E_{L U M O}^{A}-E_{\text {HOMO }}^{D}\right)-E_{B}^{C T E},
$$

where $E_{L U M O}^{A}$ is LUMO of the acceptor, $E_{H O M O}^{D}$ is HOMO of the donor, and $E_{B}^{C T E}$ is the binding energy of CTE. Since there is no clear evidence that $E_{L U M O}^{A}$ and $E_{H O M O}^{D}$ would change at the observed temperature range, a reduced $E_{B}^{C T E}$ leads to linearly increased CTE bandgap. Thus, the $V_{o c}$ would increase when $E_{B}^{C T E}$ decreases. The $E_{B}^{C T E}$ can be simply estimated with the expression of $q^{2} / 4 \pi \varepsilon_{0} \varepsilon_{r} r$, where $\varepsilon_{0}$ is the vacuum dielectric constant, $\varepsilon_{r}$ is the relative average dielectric constant of the blend, $r$ is the average initial CTE diameter after charge transfer, and $q$ is the elemental electron charge. The varied temperature should cause negligible effect on the initial CTE diameter because of very small added thermal energy compared to LUMO difference of the donor and the acceptor. Therefore, the variation of $E_{B}^{C T E}$ with temperature is dominated by $\varepsilon_{r}$ variation in the PSBTBT:PCBM blend film. Physically, it is reasonable because the increased dielectric constant of the matrix increases the screening of photogenerated electrons and holes and, thus, facilitates the charge separation.

The $\varepsilon_{r}$ was obtained at different temperatures by measuring the capacitance of the devices at $-0.1 \mathrm{~V}$. As shown in Fig. 3(b), the capacitance-frequency curves showed a plateau in the frequency range above $1 \mathrm{kHz}$ which indicates negligible influence caused by excess charge carriers. ${ }^{14}$ The variation of $\varepsilon_{r}$ was calculated by using the device capacitance at $4 \mathrm{kHz}$, although the dissociation of the CTEs happens in a very short time range of sub-picoseconds to nanoseconds. ${ }^{15}$ The reason is that the relative dielectric constant of PSBTBT:PCBM blend film measured at low frequency of $1-100 \mathrm{kHz}(\sim 4.1)$ at RT is very close to that measured in optical frequency region of $10^{11} \mathrm{kHz}(\sim 3.7$, by ellipsometry spectroscopy). It can be seen from the inset of Fig. 3(b) that device capacitance gradually increases with increasing temperature. The mechanism for the increase of dielectric constant of studied polymers is not clear yet. A possible reason is that the molecules were excited from low molecularvibrating energy states to higher molecular-vibrating energy states, leading to different molecular symmetry and polarizability. The variation of $V_{o c}$ due to the change of $E_{B}^{C T E}$ with temperature was then calculated from the following equation:

$$
\Delta V_{o c}^{T}=\frac{E_{B}^{R T}-E_{B}^{T}}{q},
$$

where $E_{B}^{T}$ is the $E_{B}^{C T E}$ at temperature $T$. By adding the calculated $\Delta V_{o c}^{T}$ from Eq. (3) to the calculated $V_{o c}^{T}$ from Eq. (1) with a fitting parameter $E_{B}^{R T}$ of $0.5 \mathrm{eV},{ }^{6,16}$ the obtained $V_{o c}$ (the red solid circle) shows a much better match with the experimentally measured values (the blue solid square) in Fig. 3(a). Since the increased temperature enhances the vibration of molecules and then affects the behavior of polarons, it is then possible that the temperature dependence of polarons also plays a role in the temperature dependence of $V_{o c}$. The polarons are actually phonon wrapped free charges after the CTE dissociation. The changed transport behavior of the polarons at elevated temperature should be included in the changed dark current and photocurrent, which contribute to the reduced $V_{\mathrm{oc}}$ with temperature and is included in the Eq. (1). Therefore, the increase of $V_{o c}$ most likely results from the temperature dependence of $E_{B}^{C T E}$.

This result can partially explain many other results about using additives to improve the PCE of BHJ-OPV (Ref. 17) and also indicates a direction to achieve better device performance through decreasing $E_{B}^{C T E}$ of the blend film.

B. Yang, J. Cox, and Y. Yuan contributed to this work equally. This work was supported in part by the Defense Threat Reduction Agency, Basic Research Award No. HDTRA1-10-1-0098 and University of Nebraska Lincoln Layman Award.

${ }^{1}$ H. Y. Chen, J. Hou, S. Zhang, Y. Liang, G. Yang, Y. Yang, L. Yu, Y. Wu, and G. Li, Nat. Photonics 3, 649 (2009).

${ }^{2}$ S. Sista, M. H. Park, Z. Hong, Y. Wu, J. Hou, W. L. Kwan, G. Li, and Y. Yang, Adv. Mater. 22, 380 (2010).

${ }^{3}$ Y. Yuan, T. J. Reece, P. Sharma, S. Poddar, S. Ducharme, A. Gruverman, Y. Yang, and J. Huang, Nat. Mater. 10, 296 (2011).

${ }^{4}$ J. Huang, G. Li, E. Wu, Q. Xu, and Y. Yang, Adv. Mater. 18, 114 (2006).

${ }^{5}$ J. Huang, G. Li, and Y. Yang, Adv. Mater 20, 415 (2008).

${ }^{6}$ T. M. Clarke and J. R. Durrant, Chem. Rev. 110, 6736 (2010).

${ }^{7}$ V. D. Mihailetchi, J. Wildeman, and P. W. M. Blom, Phys. Rev. Lett. 94, 126602 (2005).

${ }^{8}$ J. Huang, Z. Xu, and Y. Yang, Adv. Func. Mater. 17, 1966 (2007).

${ }^{9}$ C. J. Brabec, A. Cravino, D. Meissner, N. S. Sariciftci, T. Fromherz, M. T. Rispens, L. Sanchez, and J. C. Hummelen, Adv. Funct. Mater. 11, 374 (2001).

${ }^{10}$ A. Maurano, R. Hamilton, C. G. Shuttle, A. M. Ballantyne, J. Nelson, B. O'Regan, W. Zhang, I. McCulloch, H. Azimi, and M. Morana, Adv. Mater. 22, 4987 (2010).

${ }^{11}$ H. J. Snaith, N. C. Greenham, and R. H. Friend, Adv. Mater. 16, 1640 (2004).

${ }^{12} \mathrm{~K}$. Vandewal, K. Tvingstedt, A. Gadisa, O. Inganäs, and J. V. Manca, Nat. Mater. 8, 904 (2009).

${ }^{13}$ J. H. Lee, S. Cho, A. Roy, H. T. Jung, and A. J. Heeger, Appl. Phys. Lett. 96, $163303(2010)$

${ }^{14} \mathrm{~F}$. Kremer and A. Schönhals, Broadband dielectric spectroscopy (Springer, Berlin, 2003).

${ }^{15}$ J. Szmytkowski, Chem. Phys. Lett. 470, 123 (2009).

${ }^{16}$ X. Y. Zhu, Q. Yang, and M. Muntwiler, Acc. Chem. Res. 42, 1779 (2009).

${ }^{17}$ J. Peet, J. Y. Kim, N. E. Coates, W. L. Ma, D. Moses, A. J. Heeger, and G. C. Bazan, Nat. Mater. 6, 497 (2007). 